\title{
Utilisation pédagogique de la représentation graphique en classe d'anglais de spécialité
}

\section{Claudine Gardey}

\section{CpenEdition}

\section{Journals}

Édition électronique

URL : http://journals.openedition.org/asp/1641

DOI : 10.4000/asp. 1641

ISBN : 978-2-8218-0388-6

ISSN : 2108-6354

Éditeur

Groupe d'étude et de recherche en anglais de spécialité

Édition imprimée

Date de publication : 1 décembre 2002

Pagination : 137-147

ISSN : 1246-8185

\section{Référence électronique}

Claudine Gardey, «Utilisation pédagogique de la représentation graphique en classe d'anglais de spécialité », ASp [En ligne], 35-36 | 2002, mis en ligne le 26 août 2010, consulté le 19 avril 2019. URL : http://journals.openedition.org/asp/1641; DOI : 10.4000/asp.1641

Ce document a été généré automatiquement le 19 avril 2019

Tous droits réservés 


\title{
Utilisation pédagogique de la représentation graphique en classe d'anglais de spécialité
}

\author{
Claudine Gardey
}

\section{Introduction}

1 Cet article se propose de présenter une démarche élaborée par les enseignantes d'anglais de l'IUT Génie électrique et informatique industrielle et mise en place auprès des étudiants de première et seconde années de ce département. Le but de cette démarche est de familiariser les apprenants à la mise en forme visuelle de données verbales en anglais de spécialité et de leur donner ainsi les moyens de comprendre un langage verbo-visuel largement utilisé dans le monde professionnel.

\subsection{De la représentation mentale à la représentation graphique}

2 Face à un objet dont il cherche à pénétrer le sens, tout individu se construit une représentation mentale dont le degré d'organisation et d'abstraction dépend de ses connaissances antérieures, des structures cognitives qui lui sont propres et du regard qu'il porte sur la réalité. Cette architecture mentale de plus en plus complexe nous permet d'appréhender et de maîtriser progressivement le monde qui nous entoure. Les représentations peuvent revêtir une forme abstraite proche du langage (représentations propositionnelles), une forme analogique, proche de l'image, ou une combinaison de ces deux modes d'encodage (Meunier 1999). D'un point de vue fonctionnel, les images serviraient à faciliter la résolution de certains problèmes, en effectuant des comparaisons ou en permettant d'analyser des relations.

3 Pour Sallaberry (1996: 19-22), les représentations mentales sont à la fois processus et produit: processus car le sujet élabore et modifie sans cesse sa vision intérieure et produit parce que ces représentations peuvent, à un moment donné, être communiquées 
à autrui et acquérir ainsi, par le trait ou le verbe, une certaine forme, certains " contours ». En transmettant cette image, l'émetteur cherche à faire partager ses idées, mais ce faisant, il révèle aussi ce qu'il est et comment il fonctionne.

\subsection{Applications pédagogiques}

4 Si l'on considère ce qui se passe en classe de langues au cours des activités de compréhension, on s'aperçoit que la plupart des échanges verbaux ont pour fonction de renseigner le professeur sur la validité des représentations mentales de ses étudiants et sur leur capacité à en tirer des conclusions pertinentes. Le questionnement initié par l'enseignant est sans doute un moyen de guider l'apprenant vers une compréhension approfondie, mais en raison du nombre d'étudiants et de leurs difficultés d'expression, il est impossible d'évaluer à un moment donné le degré exact de compréhension de chacun. De plus, ce type d'exercice se révèle coûteux en temps et en énergie et accorde à l'enseignant une position trop centrale dans le processus didactique.

5 La technique que nous avons adoptée est fondée sur le principe "d'iconicité de la pensée » analysée par Meunier (1999). Puisque, d'après Varela «l'esprit opère par une manipulation de symboles représentant des traits du monde, ou représentant le monde comme étant dans un état certain » (1993 : 32-33), les apprenants sont incités à établir une image mentale du document à étudier et à la communiquer sous forme visuelle en mettant en évidence les relations qu'ils ont pu établir entre données nouvelles et connaissances antérieures. Le postulat sur lequel nous avons bâti notre démarche se fonde donc sur la capacité de tout être pensant à fragmenter un ensemble linéaire en éléments distincts qui seront ensuite regroupés et interconnectés pour former une structure globalisante qui puisse à la fois être immédiatement appréhendée de manière intuitive et offrir des pistes de réflexion pour des analyses plus détaillées. Les schémas, graphiques ou diagrammes ainsi créés, deviennent en quelque sorte les projections dans l'espace extérieur des modèles mentaux de leurs concepteurs. En raison de leur forme matérielle, ils se prêtent à diverses manipulations et peuvent être facilement comparés, modifiés et modélisés. Nous avions déjà abordé cette technique dans nos classes littéraires de l'enseignement secondaire (Gardey 1996 : 357-361), mais, dans le contexte de l'enseignement supérieur, nous voulions procéder à un véritable entraînement mental des étudiants afin qu'ils puissent disposer d'un outil fiable et performant qui faciliterait leur compréhension de textes d'anglais technique et général, tout en leur permettant de produire des supports visuels susceptibles d'être utilisés en milieu professionnel. Nous proposons donc dans cet article une présentation de la première phase d'apprentissage visant à la compréhension des représentations graphiques, suivie d'explications sur la mise en place d'une formation à la structuration visuelle des informations permettant d'accéder à une meilleure maîtrise de la communication non verbale.

\section{La représentation graphique, outil de médiation de la compréhension}

\subsection{Décodage et encodage de l'information}

6 La lecture, comme l'écoute, mobilise différents types d'activités cognitives: le repérage d'éléments saillants porteurs de sens, leur mise en relation et leur intégration dans un 
tout signifiant où se rejoignent connaissances antérieures et nouvelles informations. Le Ny explique que :

Comprendre le langage, c'est bien intégrer de l'information nouvelle, apportée de l'extérieur par les canaux auditifs et visuels à des représentations anciennes préexistantes, et dans le meilleur des cas à des connaissances ; c'est aussi le faire au moyen de ces représentations, utilisées comme instrument et comme source, et au moyen de procédures ou de procédés qui sont eux-mêmes de la connaissance préétablie. (1989: 228)

7 Le passage du verbal au visuel (ou scripto-visuel) suppose une mise en synergie des opérations mentales présidant au décodage des informations initiales et leur encodage sous un mode différent. Lorsque les apprenants sont invités à réorganiser un texte sous une forme personnelle et personnalisée, le choix de privilégier certaines informations au détriment d'autres données, la volonté d'établir des connexions les plus précises possibles, la capacité à adopter différents points de vue sont autant de facteurs qui gouvernent la mise en forme de leurs représentations. L'architecture mentale ainsi élaborée suit des trames plus ou moins complexes, plus ou moins abstraites selon l'intensité du travail cognitif qui aura procédé à la déconstruction reconstruction du document initial. Pour Toma :

Ces trames font ainsi apparaitre les concepts choisis parmi une gamme de possibles et surtout le calcul sémantique effectué sur ces concepts. On obtient un parcours, un réseau, dont les liens peuvent être paramétrés. L'ordonnancement des liens est, lui, caractéristique des intentions et du fonctionnement mental, onomasiologique de l'auteur. (2000: 80)

L'obligation d'avoir à mettre sous forme visuelle un ensemble de données textuelles est l'étincelle qui déclenche la mise en route du mouvement dynamique intérieur / extérieur, mais pour entretenir l'énergie cognitive nécessaire au maintien de cette dynamique, l'étudiant doit pouvoir disposer de ressources mentales efficaces, telles que des stratégies de schématisation et visualisation adaptées et variées. Il nous fallait donc mettre au point dans nos classes une démarche d'apprentissage visant à faire découvrir à l'apprenant les principes méthodologiques susceptibles de le guider dans ce double processus de délinéarisation / globalisation des informations.

\subsection{Saillance et perspective}

9 Une des richesses du langage est de pouvoir exprimer des points de vue différents à propos d'une même situation objective, par le biais d'expressions linguistiques variées. La perspective change suivant l'éclairage, c'est-à-dire l'importance accordée à l'un ou l'autre des éléments du discours (élément "saillant»). La représentation graphique utilise les multiples possibilités de l'image tout en visant à la conservation du message initial. Afin de sensibiliser les étudiants aux indispensables notions de perspective et de saillance, nous proposons l'exercice suivant, en deux parties $a$ et $b$.

Mettez sous une forme visuelle (non propositionnelle) l'énoncé suivant :

(a) John sent a letter to Susan

10 Cette première partie de l'exercice ne pose généralement aucun problème car la plupart comprennent intuitivement que la transmission de la lettre constitue l'élément saillant, et ils représentent correctement cette notion, avec plus ou moins d'étapes intermédiaires 
destinées à matérialiser le trajet de la lettre (boîte, facteur à bicyclette et même pigeon voyageur). Le schéma de la figure 1 illustre la représentation la plus courante de l'énoncé.

Figure 1. Schéma de base illustrant l'énoncé (a)

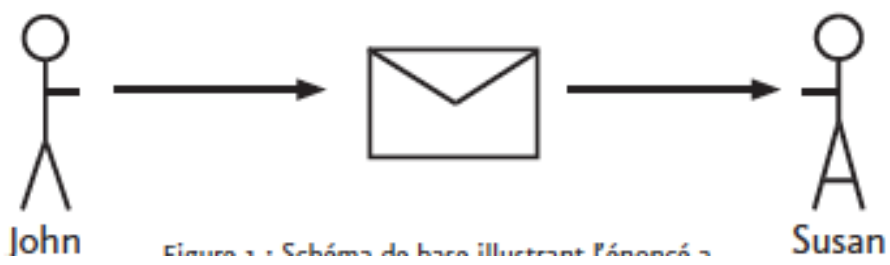

11 Ce schéma de base utilise ce que Darras (1996) appelle les "iconotypes", à savoir les résumés cognitifs graphiques les plus couramment utilisés pour dessiner êtres humains et missive. Le symbole des flèches orientées de gauche à droite bénéficie également d'un décodage instantané car fortement ancré dans notre culture occidentale européenne. Nous reviendrons plus tard sur cet aspect de l'ergonomie cognitive.

Il faut néanmoins remarquer que, pour rendre compte de l'énoncé (a), certains étudiants choisissent une forme de schéma à connotation professionnelle parfois agrémenté d'une petite note d'humour (voir figure 2).

Figure 2. Représentation de l'énoncé (a) sous forme de schéma fonctionnel

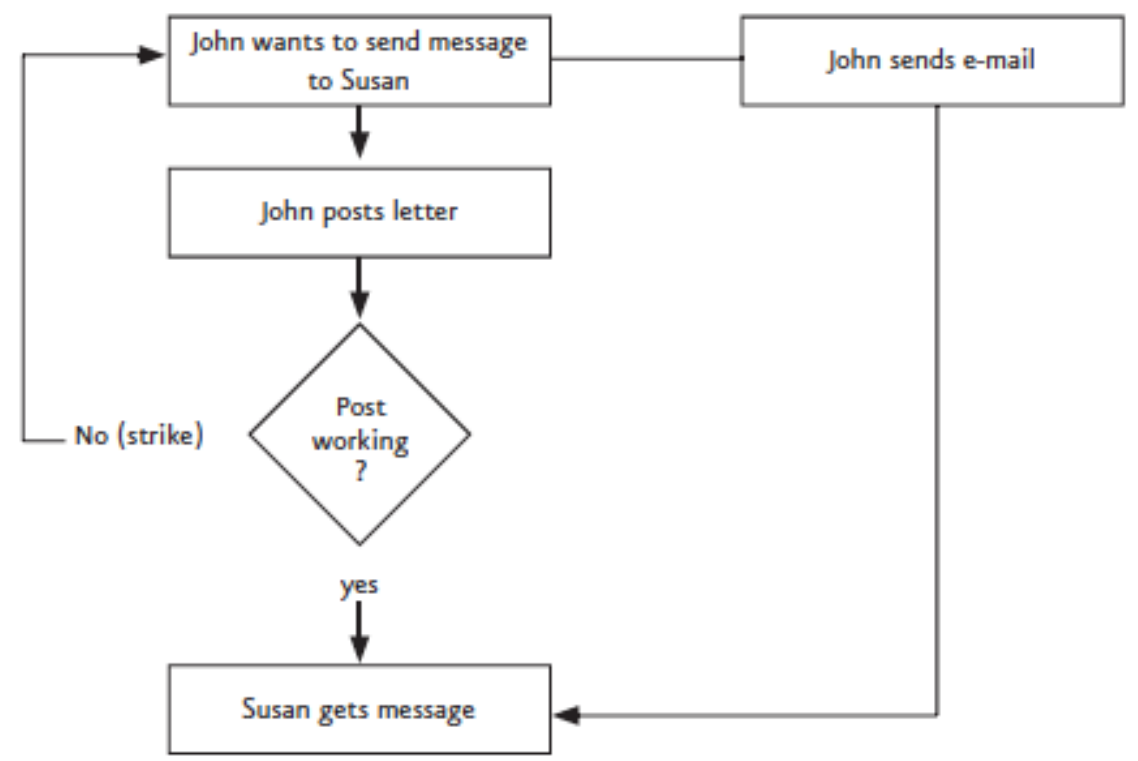

13 La lecture de ce schéma fonctionnel est moins immédiate que celle de la représentation précédente car elle fait appel à des codes visuels que les non-spécialistes en informatique ou en électronique n'interprètent qu'après réflexion (symbole du losange indiquant une prise de décision par exemple). Nous expliquerons plus loin l'importance de la culture visuelle professionnelle.

Dans la seconde partie de l'exercice, c'est l'énoncé (b) suivant qui doit être mis sous forme visuelle :

(b) John sent Susan a letter 
Si la perception de la différence est immédiate, le mot Susan ayant changé de place, la compréhension du nouveau message pose un problème à ceux qui maîtrisent mal ce point de syntaxe et les tentatives échappatoires consistant à dessiner John glissant Susan dans la boîte révèlent leur ignorance. Ceux qui ont confusément perçu le changement de perspective doivent fournir de réels efforts cognitifs pour projeter sous forme graphique leur perception mentale. La consigne d'encodage visuel les oblige à passer du percept, c'est-à-dire l'identification sommaire d'un phénomène, au concept, qui est une représentation plus abstraite et élaborée (Fortin \& Rousseau 1989). La compréhension se manifeste alors par leur décision d'accorder à l'icône Susan une place privilégiée dans leur dessin, souvent en réduisant la présence physique de John et de la lettre, ou en modifiant la disposition spatiale des trois éléments de ce triangle sémiotique. Les interrelations dans la triade, déterminées par l'émetteur concepteur du visuel, orientent ainsi l'interprétation ou reconstruction du sens de la part du récepteur.

Malgré sa simplicité apparente, cet exercice amène les apprenants à comprendre de l'intérieur ce qui est attendu d'eux: l'insight ou illumination intérieure est en effet un élément essentiel de la progression et sert de point d'ancrage référencé lorsqu'il leur est ultérieurement demandé de modifier un point de vue ou perspective.

\subsection{De l'analyse textuelle à la synthèse graphique}

La conception d'un visuel est l'aboutissement d'un travail de traitement en profondeur d'un ou plusieurs documents, qui débute par une simplification des contenus, suivie par une phase de classification et de hiérarchisation des données donnant lieu à une mise en évidence des relations et articulations (schématisation) qui débouche sur la visualisation, c'est-à-dire la traduction des informations par des variables visuelles cohérentes (Peraya 1998). Ces phases ne sont pas obligatoirement séquentielles mais peuvent s'interpénétrer et s'enrichir l'une l'autre ${ }^{1}$. De plus, à tout moment du processus, notre chien de garde intérieur ${ }^{2}$ peut intervenir pour signaler des anomalies et nous inciter à procéder à des modifications ou rectifications.

\subsubsection{Schématisation}

Si les étudiants ont été formés aux techniques de compréhension de textes et qu'ils utilisent convenablement les outils classiques de la lecture rapide que sont l'écrémage ( skimming) et la recherche ciblée (scanning), ils parviennent généralement à repérer certains mots et informations clés d'un document ne présentant pas trop de difficultés syntaxiques ou lexicales et regroupent à peu près correctement les éléments de cette sélection, opération qui fait largement appel aux réseaux sémantiques et fonctionnels intériorisés par le concepteur. Lapaire (2000: 14-16) s'appuyant sur les travaux de Johnson (1987) explique que ces structures conceptuelles préconstruites ou image schemata, ancrées dans notre vécu cognitif, socioculturel et sensori-moteur fonctionnent comme des entités composées de parties inter corrélées et servent à organiser notre expérience et notre compréhension. La complexité et le niveau d'abstraction de ces structures mentales varient grandement suivant les individus : dans l'exemple de la lettre à Susan, le schéma de base (un scripteur, une missive, une destinataire) peut être enrichi par des connexions vers d'autres schémas intériorisés (les relations épistolaires amoureuses, les grèves de la poste, le courrier électronique, etc.). Même s'ils font 
référence à des concepts socio-culturellement partagés, les regroupements effectués portent la marque personnalisée de leur concepteur qui met ainsi «en média " (Toma 2000) son univers intérieur.

L'étape suivante, la hiérarchisation des données et leur mise en relation, pose parfois problème aux étudiants qui ont des difficultés à conceptualiser, puis à attribuer une valeur relative aux concepts qu'ils ont construits. C'est pourtant ce travail cognitif intense qui contribue à faire progressivement émerger le sens en obligeant l'apprenant à effectuer des va-et-vient constants entre le document et les représentations mentales et graphiques en cours d'élaboration. La nécessité de définir la position d'une information au sein d'une organisation spatiale hiérarchisée le contraint à s'interroger sur sa fonction communicationnelle de manière plus approfondie que lors d'une simple lecture cursive. En retour, les repères conceptuels déjà mis en place lui permettent de mieux s'orienter dans sa tâche de compréhension et le guident vers des corrélations qu'il n'avait pas pressenties de prime abord. Ces interactions fonctionnelles permettent de restreindre les zones d'incertitude entourant les informations «orphelines » en les reliant utilement à des concepts en voie de formation.

Figure 3. Compréhension d'un message après une lecture cursive superficielle

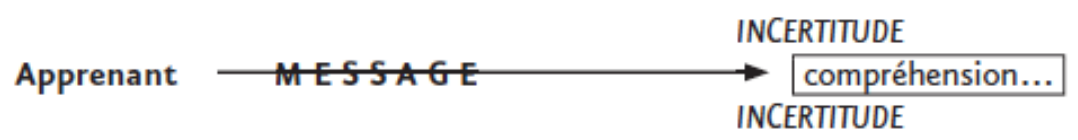

Figure 4. Compréhension d'un message par corrélation des concepts

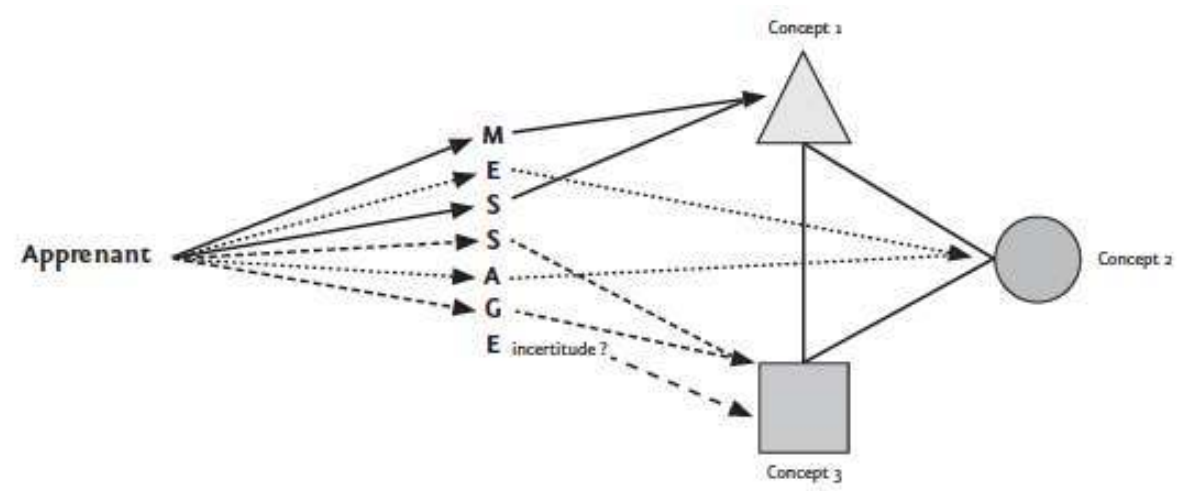

\subsubsection{Visualisation}

La phase de visualisation intervient au moment choisi par l'apprenant, au fur et à mesure du tri ou après le traitement des informations clés. Chacun est laissé libre de mener son travail comme il l'entend, mais la première attitude semble engager un travail cognitif plus complexe en raison de la distanciation nécessaire au traitement en continu de chaque information reçue en fonction du contexte sans cesse réactualisé.

Le choix du mode de représentation visuelle est révélateur de la compréhension globale du document et de la personnalité cognitive de l'étudiant. En effet, certains apprenants reconnaissant, à tort ou à raison, une situation étudiée en classe, choisissent d'emblée la forme graphique conventionnelle qui était alors associée à cette situation. D’autres 
préféreront procéder par tâtonnements avant de trouver une forme originale correspondant à leurs besoins spécifiques.

Quelques rares étudiants enfin, poussant jusqu'au bout la logique visuelle, fourniront des efforts cognitifs plus importants pour produire des métaphores visuelles qui empruntent la voie heuristique pour faire accéder instantanément le récepteur à un niveau de compréhension supérieur. L'expérience montre que ces étudiants sont généralement ceux qui ont le mieux assimilé les informations et qui les restituent oralement de la manière la plus satisfaisante, avec le recul critique qui est le signe d'un travail d'intégration en profondeur.

\section{4. Évaluation de la compréhension}

\subsubsection{Autoévaluation}

Notre but étant de conduire l'apprenant à une autonomie cognitive et langagière, nous l'encourageons à auto évaluer son travail en permanence, et donc à questionner sans cesse la validité de ses représentations. Certains a priori, c'est-à-dire des structures mentales trop prégnantes qui laissent peu de place à la flexibilité, conditionnent la compréhension et suscitent des images qui ne respectent pas le message à comprendre. L'expérience montre qu'un point de vue critique alimenté par des allers-retours fréquents entre document initial et représentations graphiques permet de repérer matériellement d'éventuels dysfonctionnements cognitifs. Il n'est pas rare de voir un étudiant qui a passé plusieurs minutes à dessiner un système complexe se rendre brusquement compte que son graphique est totalement erroné en raison de confusions lexico syntaxiques élémentaires qu'il aurait pu éviter avec un peu plus d'attention (erreurs sur behind / before ou activating / activated par exemple). Pour les étudiants peu enclins à cette activité métacognitive, la mise en route des mécanismes d'autoévaluation peut être initiée par l'enseignant avec un minimum de moyens : repérage visuel rapide des zones douteuses sur le diagramme suivi d'une injonction gestuelle à la révision des points sujets à caution. Avec un peu d'habitude, cet accompagnement cognitif silencieux ${ }^{3}$ prend à peine quelques instants par étudiant et possède un impact formateur largement supérieur à celui que pourraient avoir des explications verbales exhaustives.

\subsection{2. Évaluation extérieure}

L'évaluation d'une représentation figurative n'est pas aisée car les critères d'appréciation sont de nature plus subjective qu'objective. Les cogniticiens ont depuis longtemps modélisé des outils d'analyse des représentations sémantiques (Langacker 1987, Le Ny 1989: 75-102) mais, dans le domaine de la didactique des langues, il n'existe à notre connaissance que peu de normes établies permettant de juger de la valeur d'une transposition graphique de données verbales. L'ergonomie didactique de l'image dynamique multimédia, qui associe texte, image et son dans une dimension interactive semble faire l'objet de plus nombreuses recherches que la mise en média statique. Les critères d'évaluation que nous avons retenus dans le contexte de notre recherche sont les suivants :

- Le degré de validité : le message transmis par le visuel correspond-t-il au message textuel? Existe-t-il des erreurs flagrantes de transposition graphique dues à des problèmes de 
compréhension qui gauchissent le sens du contenu initial au point de le rendre non conforme?

- Le degré de schématicité (Langacker 1987) : ce point concerne l'adéquation entre le contenu initial et sa mise en forme visuelle. Le schéma respecte-t-il la fonction communicationnelle du document de départ? Les relations fondamentales (inclusion, séparation, identité et association) ont-elles été respectées? Remarque-t-on des efforts de symbolisation ou de mise en relation métaphorique?

- Le degré de scientificité (Toma 2000) : les domaines de connaissances scientifiques explorés par le concepteur du visuel sont-ils maîtrisés et approfondis? Des concepts corrélés sont-ils évoqués?

- Le degré de didacticité (Toma 2000) : la visualisation des concepts fait-elle apparaître des trames discursives faciles à interpréter ? Les éléments saillants sont-ils immédiatement perceptibles? Les mises en relation sont-elles explicites et les transitions entre événements cognitifs assez claires pour ne pas gêner la lecture de la chaîne explicative ?

- Le degré d'esthétisme (Peraya 1998) : le visuel est-il agréable à regarder ? Procure-t-il des sensations esthétiques qui encouragent et facilitent son décryptage sémantique?

Si le premier de ces critères (validité) peut donner lieu à des évaluations d'ordre quantitatif grâce à des marqueurs de compréhension identifiables et mesurables d'après un barème pré-établi ${ }^{4}$, les appréciations obtenues en fonction des autres critères sont plutôt d'ordre qualitatif et dépendent $d u$ potentiel communicationnel des représentations graphiques, plus complexe à évaluer objectivement.

\section{La représentation graphique, outil de médiation de la communication}

\subsection{La co-construction de sens}

La phase d'encodage graphique, réalisée seul ou à deux, est suivie d'une phase de mise en commun des productions. Quelques étudiants dessinent rapidement leur schéma au tableau et le commentent en anglais. La disposition communicative de la salle d'anglais, avec plusieurs tableaux répartis le long des murs, autorise une vision simultanée des diverses propositions et encourage la comparaison et la critique dans la langue cible. Chacun défend son point de vue, propose des améliorations ou suggère des modifications afin de parvenir à la création collective de modèles graphiques satisfaisants qui serviront de supports à des exercices ultérieurs d'expression ou de rappel mnémonique. Dans cette activité socialement orientée, les transactions verbales servent à la résolution de situations problématiques et la langue-cible est utilisée comme un outil de négociation nécessaire à la réalisation d'un objectif commun.

De nombreux auteurs (Perret-Clermont 1975, Doise 1982, Ghiglione et al. 1990) ont souligné la dimension structurante des interactions sociales en situation d'apprentissage. La confrontation des représentations permet au groupe classe de négocier une référence coconstruite au sein d'un espace interlocutoire (Ghiglione et al. 1990 : 176-226) où s'établit une compréhension commune nourrie de certitudes, de doutes et d'oppositions. En participant à l'élaboration d'une représentation consensuelle, l'étudiant apprend à réorganiser ses concepts et à enrichir ses connaissances. De plus le rappel mnémonique d'un modèle est plus facile que celui d'un discours en raison du travail cognitif plus intense qui accompagne sa structuration. Le support visuel devient alors une aide 
précieuse dans cette activité si redoutée qu'est l'exposé oral, en limitant le recours systématique à des notes écrites et en permettant à l'orateur de s'entraîner à ponctuer son discours d'une gestuelle adaptée.

\subsection{Les codes de communication professionnelle}

28 Parce qu'il est un outil de médiation qui facilite la mise en relation et l'intégration des données, le vecteur visuel joue un rôle important dans les communications scientifiques, mais le décodage des schémas n'est rapide et efficace que si l'auditoire partage la même culture et possède un degré d'expertise suffisant pour comprendre des codes graphiques hautement spécialisés. Une sensibilisation aux conventions visuelles de la spécialité est donc nécessaire pour développer chez les étudiants des habitudes mentales qui leur seront utiles au cours de leur vie professionnelle.

Rowley-Jolivet (2000 : 133-154) a analysé en détail les différents aspects de la visualisation professionnelle et nous nous sommes appuyée sur certaines conclusions de ses recherches pour déterminer nos priorités d'enseignement / apprentissage et former nos étudiants à l'imagerie de leur discipline. Le schéma fonctionnel de la Figure 2 illustre d'ailleurs cette imprégnation disciplinaire chez un étudiant en électronique et informatique industrielle. D'autres modèles, tels les schémas systémiques ou le diagramme d'analyse des causes d'Hishikawa, sont présentés à des spécialistes d'autres disciplines. Il va sans dire que les outils langagiers nécessaires à l'explicitation verbale de tels schémas font l'objet d'une étude approfondie en classe.

\subsection{L'ergonomie cognitive}

30 Nous avons vu plus haut (Lapaire 2000, Johnson 1996, Varela et al. 1993) que les êtres humains possèdent des structures cognitives préconstruites ancrées dans notre expérience sensori-motrice. Ainsi les notions de bas et de haut, d'intérieur et d'extérieur conditionnent par exemple notre compréhension du monde. De plus nos habitudes culturelles nous font attribuer certaines valeurs à nos cheminements oculaires lors de la lecture de visuels : ainsi le sens gauche-droite est fortement corrélé aux notions de chronologie ou de causalité.

31 Les apprenants ne respectent pas toujours les habitudes cognitives de leur auditoire et la lisibilité de leurs productions graphiques en est grandement affectée. Lorsqu'ils commentent leur schéma, leur discours semble ne pas être en "synchronisation » avec l'information visuelle (Rowley-Jolivet 2000 : 146) ce qui crée des confusions et perturbe la compréhension. Une réflexion sur la complémentarité du canal verbal et du canal visuel leur permet de progresser dans la maîtrise de la dimension picturale ${ }^{5}$.

Un autre aspect de l'ergonomie visuelle nous paraît également intéressant. Nous avons remarqué que, familiarisé à la lecture des cartes conceptuelles de navigation proposées par l'hypermédia en tant que substitut ouvert et dynamique des plans traditionnels à conception linéaire, l'esprit de l'étudiant lambd@ semble devenir de plus en plus réceptif à des modes de mise en relation réticulaires. Cette tendance est particulièrement visible dans la présentation des plans au début des exposés, qui négligent la disposition linéaire classique au profit d'une distribution spatiale en réseau.

On note aussi que les étudiants intéressés par les nouvelles technologies s'habituent rapidement aux codes spécifiques de l'imagerie électronique et acquièrent, à des niveaux 
plus ou moins élevés, une compétence visuelle influencée par la culture Internet, perceptible dans leur façon de «mettre en scène » l'information sur support statique ou dynamique : organisation spatiale accordant des valeurs différentes à la moitié droite ou à la moitié gauche du support, options chromatiques ou typographiques, insertion de commentaires humoristiques iconiques ou sonores, etc. Il nous semble assister ici à l'émergence d'une sémiotique particulière caractérisée par une redéfinition progressive de la syntaxe visuelle.

Le code couleur est un autre point sur lequel nous portons notre attention. Aux premiers temps de l'utilisation du numérique, les mille et une couleurs proposées par l'ordinateur étaient utilisées uniquement pour leur esthétisme ou leur aspect racoleur. Nous constatons depuis peu de temps une prise de distance réfléchie vis-à-vis de l'utilisation de la couleur qui sert maintenant à marquer liens et oppositions ou à nuancer le message d'une coloration affective ${ }^{6}$. Cette tendance indique-t-elle une réelle prise en compte de la couleur comme vecteur de communication graphique? Les moyens de reprographie modernes joueraient alors un rôle dans l'affaiblissement progressif de la charge cognitive du tout noir et blanc.

\section{Conclusion}

Le monde professionnel utilise largement l'interface analogique (dessins, tableaux, schémas) pour mettre en scène des concepts complexes et aider à leur compréhension. L'introduction d'une phase de restitution graphique des données verbales en classe d'anglais de spécialité nous semble donc justifiée dans la mesure où elle

- engage l'étudiant dans des activités cognitives de haut niveau qui accélèrent l'intégration des informations ;

- familiarise le futur spécialiste avec les codes de communication visuelle de sa discipline ;

- lui enseigne quelques notions de base d'ergonomie visuelle ;

- encourage la co-construction de sens par la confrontation des représentations ;

- facilite la prise de parole et le rappel mnémonique des connaissances.

Nous partageons les conclusions de Landois-Maynard (2000: 422-423) sur la facilité de mise en place de cet outil didactique qui se révèle être à la fois souple et rigoureux, méthodique et polyvalent, et dont nous ne soupçonnions pas la richesse didactique avant d'en systématiser l'usage dans notre enseignement.

Il reste bien sûr beaucoup à faire, surtout en ce qui concerne l'évaluation quantifiée de l'impact de cette technique sur la compréhension et la mémorisation. De plus, les possibilités offertes par l'informatique nous incitent à ne plus nous limiter à l'imagerie statique, mais à faire explorer aux apprenants l'immense potentiel de l'«idéographie dynamique " (Levy 1991) et à mettre en interaction toutes leurs ressources cognitives pour imag...iner de nouveaux modes de mises en multimédia de l'information. 


\section{BIBLIOGRAPHIE}

Darras, Bernard. 1996. Au commencement était l'image. Du dessin de l'enfant à la communication de l'adulte. Paris : ESF.

Doise, Wilhem. 1982. L'Explication en psychologie sociale. Paris : Presses Universitaires de France. Fortin, C. et R. Rousseau. 1989. Psychologie cognitive. Québec : Presses de l'Université de Québec. Gardey, Claudine. 1996. «Interactions et construction du savoir en classe de langues ». Thèse de doctorat, Université Bordeaux 2, sous la direction de M. Perrin.

Gattegno, Caleb. 1972. Teaching Foreign Languages in Schools: The Silent Way. New York : Educational Solutions.

Gattegno, Caleb. 1976. Common Sense in Language Teaching. New York : Educational Solutions.

Ghiglione, Rodolphe, Claude Bonnet et Jean-François Richard. 1990. Cognition, représentation, communication, vol. 3. Paris : Dunod.

Johnson, Mark. 1996. The Body in the Mind. The Bodily Basis of Meaning, Imagination and Reason. Chicago : University of Chicago Press.

Landois-Maynard, Sylvie. 2000. « De l'image invisible au texte révélé ». ASp 27-30, 411-423.

Langacker, R. 1987. Foundations of Cognitive Grammar, vol.1. Stanford, CA : Stanford University Press.

Lapaire, Jean-Rémi. 2000. « Got the picture? Image, image schemata, imaginative reason ». ASp 27-30, 7-17.

Le Ny, Jean-François. 1989. Science cognitive et compréhension du langage. Paris : Presses Universitaires de France.

Levy, Pierre. 1991. L'Idéographie dynamique. Vers une imagination artificielle?. Paris : La Découverte. Meunier, Jean-Pierre. 1999. « Connaître par l'image ». Janvier 2001 http://www.comu.ucl.ac.be/ reco/grems/jpweb/rec10/connaitre.htm.

Peraya, Daniel. 1998. « La Visualisation de l'information ». Février 2001 http://tecfa.unige.ch/ themes/tdsr/visuali/manuel/visuali-man-visuali-Un.html.

Perret-Clermont, Anne-Nelly. 1975. La Construction de l'intelligence dans l'interaction sociale. Berne : Peter Lang.

Rowlet-Jolivet, Elizabeth. 2000. «Image as text. Aspects of the shared visual language of scientifi c conference participants ». ASp 27-30, 133-154.

Sallabery, Jean-Claude. 1996. Dynamique des représentations dans la formation. Paris : L'Harmattan. Toma, Tony. 2000. « Avancées dans le pré-verbal : les trames discursives conceptuelles ». ASp 27-30, 63-91.

Varela, Francisco. 1993. «L'auto-organisation : de l'apparence au mécanisme ». In Dumouchel P. et J.-P. Dupuy (dir.), L'Auto-organisation de la physique au politique, colloque de Cerisy. Paris : Seuil. 
Varela, Francisco, Evan Thompson et Eleanor Rosch. 1993. L'Inscription corporelle de l'esprit. Paris : Éditions du Seuil.

\section{NOTES}

1. Le choix, inconscient ou non, d'accomplir la première phase du processus avant d'aborder la seconde et la troisième (stratégie de type analytique ou sérialisante) ou de mener de front analyse des détails et ébauche d'une structuration visuelle globale (stratégie globalisante) dépend en grande partie du profil d'apprentissage de l'apprenant et de ses stratégies cognitives préférentielles (Gardey 1996 : 177-205).

2. Le chien de garde ou watchdog est un terme emprunté au vocabulaire de l'électronique. Il s'agit d'un sous-système destiné à repérer et réguler les dysfonctionnements. Ce terme définit assez bien les principes mis en œuvre dans l'auto-évaluation.

3. Sans en adopter tous les principes, nous avons souvent introduit avec succès dans nos classes certaines techniques inspirées de l'approche du Silent Way de Gattegno (1972). En effet, le silence volontaire de l'enseignant, parfois ponctué de rares gestes indicatifs, renvoie l'apprenant à l'examen de ses propres représentations et l'amène donc à en reconsidérer la validité et à imaginer de nouvelles approches.

4. La restitution intégrale d'un schéma incomplet par le biais de données écrites ou orales constitue un exercice d'évaluation de la compréhension fiable et facile à noter si les indices à restituer sont affectés du nombre de points correspondant à l'absence, à la présence ou au placement incorrect des informations clés dans l'organisation finale.

5. Lors des stages en milieu professionnel, il est de plus en plus demandé à nos étudiants de réaliser des interfaces graphiques machine-utilisateur. Il nous semble donc essentiel qu'ils aient pu intégrer quelques notions d'ergonomie cognitive au cours de leurs études

6. Voir l'étude des rapports entre couleur et cognition dans Varela (1993 : 212-233).

\section{RÉSUMÉS}

Cet article traite de l'utilisation pédagogique de la mise en forme visuelle de données verbales en classe d'anglais de spécialité. Les représentations graphiques, très présentes dans les communications scientifiques, sont des vecteurs essentiels de la médiation du sens en milieu professionnel. Il nous semble donc important de développer chez les étudiants les habitudes cognitives nécessaires à la structuration visuelle de l'information. L'expérience montre que cette médiation du sens par l'image facilite les apprentissages à tous les niveaux du processus didactique.

The aim of this paper is to explore the pedagogical dimensions of the visual organization of verbal data in the ESP classroom. Now widely used in scientific communication, visuals are effective devices for the mediation of meaning. It is therefore important to train students to structure information through the use of mental and pictorial representations. Experience suggests that the visual-verbal interplay facilitates learning at all levels of the didactic process. 
INDEX

Mots-clés : médiation du sens, représentation graphique, représentation mentale, structure cognitive

Keywords : cognitive structure, mediation of meaning, mental representation, pictorial representation

\section{AUTEUR}

\section{CLAUDINE GARDEY}

Claudine Gardey est maître de conférences agrégée d'anglais à l'Université Bordeaux 1, à l'IUT A (département Génie électrique et Informatique industrielle). Elle participe également à la formation continue des professeurs d'anglais de l'enseignement secondaire (classique et technique) dans le cadre de l'IUFM. Ses recherches portent sur les aspects cognitifs de l'enseignement/apprentissage des langues, la didactique de l'anglais et les nouvelles technologies. claudine.gardey@u-bordeaux1.fr 